\title{
Restriction of Food Intake Prevents Postinfarction Heart Failure by Enhancing Autophagy in the Surviving Cardiomyocytes
}

Takatomo Watanabe, ${ }^{*}$ Genzou Takemura, ${ }^{* \dagger}$ Hiromitsu Kanamori, ${ }^{*}$ Kazuko Goto, ${ }^{*}$ Akiko Tsujimoto, ${ }^{*}$ Hideshi Okada, * Itta Kawamura, ${ }^{*}$ Atsushi Ogino, ${ }^{*}$ Toshiaki Takeyama, ${ }^{*}$ Tomonori Kawaguchi, ${ }^{*}$ Kentaro Morishita, ${ }^{*}$ Hiroaki Ushikoshi, ${ }^{*}$ Masanori Kawasaki, ${ }^{*}$ Atsushi Mikami, ${ }^{*}$ Takako Fujiwara, ${ }^{\ddagger}$ Hisayoshi Fujiwara, ${ }^{\S}$ and Shinya Minatoguchi*

From the Department of Cardiology, * Gifu University Graduate School of Medicine, Gifu; the Department of Internal Medicine, ${ }^{\dagger}$ Asahi University School of Dentistry, Mizuho; the Department of Food and Nutrition, ${ }^{\ddagger}$ Sonoda Women’s University, Amagasaki; and the Hyogo Prefectural Amagasaki Hospital, ${ }^{\S}$ Amagasaki, Japan

Accepted for publication January 3, 2014.

Address correspondence to Genzou Takemura, M.D., Ph.D., Department of Internal Medicine, Asahi University School of Dentistry, 1851 Hozumi, Mizuho, Gifu 5010296, Japan. E-mail: gt@dent. asahi-u.ac.jp.

\begin{abstract}
We investigated the effect of restriction of food intake, a potent inducer of autophagy, on postinfarction cardiac remodeling and dysfunction. Myocardial infarction was induced in mice by left coronary artery ligation. At 1 week after infarction, mice were randomly divided into four groups: the control group was fed ad libitum (100\%); the food restriction (FR) groups were fed $80 \%, 60 \%$, or $40 \%$ of the mean amount of food consumed by the control mice. After 2 weeks on the respective diets, left ventricular dilatation and hypofunction were apparent in the control group, but both parameters were significantly mitigated in the FR groups, with the $60 \%$ FR group showing the strongest therapeutic effect. Cardiomyocyte autophagy was strongly activated in the FR groups, as indicated by up-regulation of microtubule-associated protein 1 light chain 3-II, autophagosome formation, and myocardial ATP content. Chloroquine, an autophagy inhibitor, completely canceled the therapeutic effect of FR. This negative effect was associated with reduced activation of AMP-activated protein kinase and of ULK1 (a homolog of yeast Atg1), both of which were enhanced in hearts from the FR group. In vitro, the AMPactivated protein kinase inhibitor compound C suppressed glucose depletion-induced autophagy in cardiomyocytes, but did not influence activity of chloroquine. Our findings imply that a dietary protocol with FR could be a preventive strategy against postinfarction heart failure. (Am J Pathol 2014, 184: 1384-1394; http://dx.doi.org/10.1016/j.ajpath.2014.01.011)
\end{abstract}

Large myocardial infarctions lead to severe chronic heart failure, with adverse remodeling of the left ventricle characterized by cavity dilatation and diminished cardiac performance. ${ }^{1}$ The magnitude of the acute infarction, determined within several hours of an attack, ${ }^{2}$ is the most critical determinant of subsequent ventricular remodeling and heart failure. Cardiac remodeling is a complicated process, however, and many other factors are also associated with disease progression during the chronic stage after infarction. These include hypertrophy, degeneration, or late death of cardiomyocytes; fibrosis; expression of various cytokines; and the dynamics of noncardiomyocytes within the infarct tissue. ${ }^{3-5}$

Recent studies also provide evidence of autophagy in the affected cardiomyocytes. ${ }^{6,7}$ Autophagy is a physiological process in which a cell digests its own constituents, including subcellular organelles, via the lysosomal degradative pathway. The function of autophagy is in large part survival oriented. It is activated under both basal conditions and conditions of stress (eg, starvation) as degraded membrane lipids and proteins within autophagosomes are recruited to maintain needed levels of ATP production and protein synthesis, thereby promoting cell survival. ${ }^{8}$ However, autophagy

\footnotetext{
Supported in part by grants-in-aid for scientific research from the Ministry of Education, Science, and Culture of Japan (H.K.), a grant-in-aid from the Japanese Medical Association (G.T.), and a research grant from Gifu University (S.M.).

Disclosures: None declared.
} 
is also a mode of cell death that occurs during tissue and organ development to eliminate unnecessary cells. ${ }^{9}$ In the heart, autophagy has been shown to be an adaptive response that protects the myocardium from hemodynamic overload and acute ischemic death. ${ }^{10,11}$ Autophagy occurs constitutively within the normal myocardium, but it is substantially increased in cases of ischemic cardiomyopathy, heart failure, and cardiac hypertrophy. ${ }^{6,7,12,13}$ The functional role of autophagy in heart disease (ie, whether it mediates cell survival or cell death) and whether it up-regulates or downregulates cellular function remain poorly understood. ${ }^{12,14}$

Using a mouse model of myocardial infarction, we recently observed activated autophagy in surviving cardiomyocytes during both subacute and chronic stages of a large myocardial infarction (ie, 1 and 3 weeks after infarction, respectively). In addition, we have found that, although inhibiting this autophagy exacerbates postinfarction cardiac remodeling, enhancing it mitigates the remodeling. ${ }^{15}$ We had previously used rapamycin to accelerate autophagy, but administration of rapamycin for long periods could be harmful if applied clinically, because of its inhibitory effect on cell growth. ${ }^{15-17}$ Starvation, on the other hand, is also a potent inducer of cardiac autophagy, and inhibition of starvation-induced autophagy causes heart failure in adult mice. ${ }^{18,19}$ Caloric restriction was reported to stimulate autophagy to mediate some beneficial effects in the heart. ${ }^{20} \mathrm{We}$ therefore hypothesized that regulated restriction of food intake might augment autophagy to exert beneficial effects on postinfarction cardiac remodeling and dysfunction. In the present study, we restricted food intake to various degrees in mice after large myocardial infarctions and examined both the therapeutic effects and possible pathogenic effects.

\section{Materials and Methods}

\section{Animals and Experimental Protocols}

This study conforms to the Guide for the Care and Use of Laboratory Animals published by the NIH (publication 85-23, revised 1996) and was approved by the Institutional Animal Research Committee of Gifu University. Mice were initially anesthetized using $2 \%$ halothane in a mixture of $\mathrm{N}_{2} \mathrm{O}$ and $\mathrm{O}_{2}$ (each at $0.5 \mathrm{~L} /$ minute) administered via a nasal mask. The mice were then intubated with a 20 -gauge intravenous catheter and ventilated with $0.5 \%$ halothane in a mixture of $\mathrm{N}_{2} \mathrm{O}(0.1 \mathrm{~L} /$ minute $)$ and $\mathrm{O}_{2}(0.5 \mathrm{~L} /$ minute $)$ using a rodent ventilator. Myocardial infarction was induced in male C57BL/6J mice (CLEA Japan, Fuji, Japan) at 10 weeks of age by ligating the left coronary artery, as described previously. ${ }^{21,22}$ Sham-treated animals underwent the same surgical procedures except that the left coronary artery was not occluded.

Echocardiographic examination was performed on surviving mice at 1 week after coronary artery ligation $(n=29)$. Randomly selected mice $(n=6)$ were allowed free access to food (control group), and the average caloric intake was calculated from the daily food intake over a 2 -week period. This was regarded as $100 \%$ caloric intake. The remaining mice were assigned to food restriction (FR) groups with food intake at $80 \%(n=6), 60 \%(n=8)$, or $40 \%(n=9)$ of the average caloric intake for 2 weeks.

In another set of experiments, mice surviving at 1 week after coronary artery ligation $(n=24)$ were assigned, after echocardiographic examination, to saline treatment (control) $(n=12)$ or to treatment with $10 \mathrm{mg} / \mathrm{kg}$ chloroquine (Sigma-Aldrich, St. Louis, MO) $(n=12)$. Treatments were administered for 2 weeks using subcutaneously embedded osmotic minipumps (ALZET micro-osmotic pump 1002; DURECT, Cupertino, CA). Chloroquine is thought to suppress inflammation by raising lysosomal $\mathrm{pH}$, thereby inhibiting lysosomal activity. ${ }^{23,24}$ The $10 \mathrm{mg} / \mathrm{kg}$ dose of chloroquine is reported to suppress autophagy in the mouse heart without any apparent adverse effects. ${ }^{25}$ To assess the effects of these treatments in mice without infarction, the saline or chloroquine was administered in the same manner to sham-operated mice at 1 week after surgery $(n=3$ per group), and all mice were examined at 2 weeks after the start of treatment (ie, 3 weeks after surgery).

\section{Physiological Studies}

Echocardiography and cardiac catheterization were performed as described previously. ${ }^{21,22}$ Because of its invasiveness, cardiac catheterization was performed only just before sacrifice.

\section{Histology}

Once the physiological measurements were complete, the mice were sacrificed. The hearts were removed, weighed, and cut into transverse slices at the mid-papillary muscle level. They were then fixed in $10 \%$ buffered formalin, embedded in paraffin, cut into 4- $\mu \mathrm{m}$-thick sections and stained with H\&E and Masson's trichrome. Cardiomyocyte size, expressed as the transverse diameter of myocytes cut at the level of the nucleus, was assessed in 20 randomly chosen high-power fields (HPFs) $(\times 600)$ in each section.

\section{Immunohistochemistry}

After deparaffinization, 4- $\mu$ m-thick sections were incubated with a primary antibody against microtubule-associated protein-1 light chain 3 (LC3; MBL Medical \& Biological Laboratories, Nagoya, Japan). A Vectastain Elite ABC kit (Vector Laboratories, Burlingame, CA) was then used to immunostain the sections; diaminobenzidine served as the chromogen, and nuclei were counterstained with hematoxylin. Quantitative assessments, including the number of immunopositive cells or immunopositive dots within cells, were performed in 20 randomly selected HPFs $(\times 600)$ using a multipurpose color image processor (Nireco, Tokyo, Japan). The border area was defined as the area within a HPF that contains both infarcted 
and surviving myocardium, and the remote area was defined as the myocardial region without any infarction.

\section{Electron Microscopy}

Cardiac tissue was quickly cut into 1-mm cubes, immersionfixed in $2.5 \%$ glutaraldehyde in $0.1 \mathrm{~mol} / \mathrm{L}$ PBS $(\mathrm{pH} 7.4)$ overnight at $4{ }^{\circ} \mathrm{C}$, and postfixed in $1 \%$ buffered osmium tetroxide. The specimens were then conventionally processed and examined under an electron microscope (H-800; Hitachi, Tokyo, Japan).

\section{Western Blotting}

Proteins $(20 \mu \mathrm{g})$ extracted from hearts $(n=3$ to 6 hearts per group) were subjected to $10 \%$ or $15 \%$ PAGE and then were transferred onto polyvinylidene difluoride membranes. The membranes were probed using the following primary antibodies: LC3 and p62 (MBL); cathepsin D (Santa Cruz Biotechnology, Dallas, TX); AMP-activated protein kinase (AMPK) and phosphorylated AMPK (p-AMPK) (Cell Signaling Technology, Danvers, MA); ULK1 (sc-10900; Santa Cruz Biotechnology) and p-ULK1 (6888; Cell Signaling Technology); Akt and p-Akt (Cell Signaling Technology); Sirt1 (Millipore, Billerica, MA), and histone $\mathrm{H} 3$ and acetylated histone H3 (Calbiochem; Millipore). Blots were then visualized using enhanced chemiluminescence (Amersham; GE Healthcare, Little Chalfont, UK). $\alpha$-Tubulin (analyzed using an antibody from Santa Cruz Biotechnology) served as the loading control.

\section{Myocardial ATP Content}

Myocardial ATP content was measured using an ATP bioluminescence assay kit (TOYO Ink Group, Tokyo, Japan) according to the manufacturer's instructions. Absorbance was measured using a GloMax 20/20n luminometer (Promega,
Madison, WI). Experiments were performed in triplicate for each group.

\section{In Vitro Study}

Cardiomyocytes were isolated from 1-day-old neonatal C57BL/6J mice as previously reported. ${ }^{26,27}$ The cells were then plated in laminin-coated slide glass chambers and incubated in Dulbecco's modified Eagle's medium (SigmaAldrich) containing $10 \%$ fetal bovine serum (Sigma-Aldrich) and penicillin/streptomycin at $37^{\circ} \mathrm{C}$ in a $\mathrm{CO}_{2}$ incubator $(95 \%$ air $/ 5 \% \mathrm{CO}_{2}$ ). At 2 days after plating, the medium was replaced with fetal bovine serum-free Dulbecco's modified Eagle's medium (control group), or with fetal bovine serum-free and glucose-free Dulbecco's modified Eagle's medium (Gibco 0983; Life Technologies, Carlsbad, CA) in which glucose was replaced with $11 \mathrm{mmol} / \mathrm{L}$ mannitol (starvation group), or with fetal bovine serum-free Dulbecco's modified Eagle's medium containing $3 \mu \mathrm{mol} / \mathrm{L}$ chloroquine for 4 hours (chloroquine-treated group). Each group was then treated with vehicle or $20 \mu \mathrm{mol} / \mathrm{L}$ of the AMPK inhibitor compound C (Calbiochem; Millipore) ${ }^{28}$ Four hours later, cells were subjected to Western blotting for AMPK, p-AMPK, ULK1, p-ULK1, and $\beta$-actin (Sigma-Aldrich), or to immunofluorescence assay for LC3 using anti-LC3 antibody as the primary antibody followed by Alexa Fluor 488 (green; Life Technologies) labeling and Hoechst 33342 counterstain analyzed under a fluorescence microscope (BZ-8000; Keyence, Osaka, Japan), or to measurement of ATP content. Experiments were performed in triplicate for each group.

\section{Statistical Analysis}

Significance of differences between groups was evaluated using one-way analysis of variance with a post hoc Newman-Keuls multiple comparisons test. $P<0.05$ was considered significant. Data are expressed as means $\pm \mathrm{SEM}$.

Table 1 Food Intake, Body Weight, Heart Weight, and Heart Weight/Body Weight Ratio over Time, by Treatment Group

\begin{tabular}{|c|c|c|c|c|c|}
\hline & $\begin{array}{l}\text { Sham surgery } \\
(n=6)\end{array}$ & $\begin{array}{l}\text { Control } \\
(n=6)\end{array}$ & $\begin{array}{l}80 \% \text { FR } \\
(n=6)\end{array}$ & $\begin{array}{l}60 \% \text { FR } \\
(n=8)\end{array}$ & $\begin{array}{l}40 \% \text { FR } \\
(n=9)\end{array}$ \\
\hline \multicolumn{6}{|c|}{ Food intake (g/day) } \\
\hline Week 1 & $3.81 \pm 0.3$ & $3.44 \pm 0.5$ & 2.8 & 2.1 & 1.4 \\
\hline Week 2 & $3.69 \pm 0.1$ & $3.70 \pm 0.1$ & 3.0 & 2.2 & 1.5 \\
\hline Week 3 & $3.71 \pm 0.2$ & $3.74 \pm 0.3$ & 3.0 & 2.2 & 1.5 \\
\hline \multicolumn{6}{|c|}{ Body weight (g) } \\
\hline 0 days & $24.0 \pm 0.9$ & $23.7 \pm 0.9$ & $24.3 \pm 1.8$ & $24.5 \pm 1.4$ & $24.1 \pm 1.9$ \\
\hline 3 weeks & $26.4 \pm 0.9$ & $25.9 \pm 1.3$ & $19.9 \pm 0.8^{* \dagger}$ & $18.6 \pm 0.9^{* \dagger}$ & $16.7 \pm 1.2^{* \dagger \ddagger \S}$ \\
\hline \multicolumn{6}{|c|}{ Heart weight (mg) } \\
\hline 3 weeks & $91.7 \pm 4.1$ & $138.8 \pm 21.7^{*}$ & $94.0 \pm 7.0^{\dagger}$ & $83.0 \pm 4.8^{\dagger}$ & $89.0 \pm 8.8^{\dagger}$ \\
\hline \multicolumn{6}{|c|}{ Heart weight/body weight $(\mathrm{mg} / \mathrm{g})$} \\
\hline 3 weeks & $3.5 \pm 0.2$ & $5.4 \pm 0.9^{*}$ & $4.7 \pm 0.4^{*}$ & $4.5 \pm 0.3^{* \dagger}$ & $5.4 \pm 0.6^{\star \S}$ \\
\hline
\end{tabular}




\section{Results}

\section{Food Restriction}

When fed ad libitum (100\%), the mice consumed $3.44 \pm 0.5 \mathrm{~g}$ food per day during week 1 after myocardial infarction, $3.70 \pm 0.6 \mathrm{~g}$ during week 2 , and $3.74 \pm 0.3 \mathrm{~g}$ during week 3 $(n=3)$. Food restriction was started at 1 week after infarction. Thus, the mice receiving $80 \%, 60 \%$, and $40 \%$ of the food consumed by the control group ( $80 \%$ FR, $60 \% \mathrm{FR}$, and $40 \% \mathrm{FR}$ groups, respectively) were fed $3.0 \mathrm{~g} / \mathrm{day}, 2.2 \mathrm{~g} / \mathrm{day}$, or $1.5 \mathrm{~g} / \mathrm{day}$, respectively, during weeks 2 and 3 after infarction (Table 1).

\section{Effect of FR on Postinfarction Cardiac Function and Remodeling}

Echocardiography and cardiac catheterization performed at 3 weeks after infarction revealed marked left ventricular remodeling and dysfunction in the control group, compared with the sham-surgery group (Figure 1). However, these adverse effects were mitigated in the FR groups, with the $60 \%$ FR group showing the maximal benefit (Figure 1). Histological sections showed that cavity dilatation was diminished in the FR groups (Figure 2A), and although body weights declined in proportion to the degree of FR, the heart weight/body weight ratios were nonetheless smallest in the $60 \%$ FR group and were smaller in the all FR groups than in the control group (Table 1). The size of the myocardial infarct (based on the percent segmental length) was also smaller in the FR groups and smallest in the 60\% FR group (Figure 2). In the FR groups, cardiomyocyte hypertrophy (based on the mean diameter of cardiomyocytes) was attenuated in the border areas and in areas remote from the infarct, and this tendency was most conspicuous in the $60 \%$ FR group (Figure 2B).

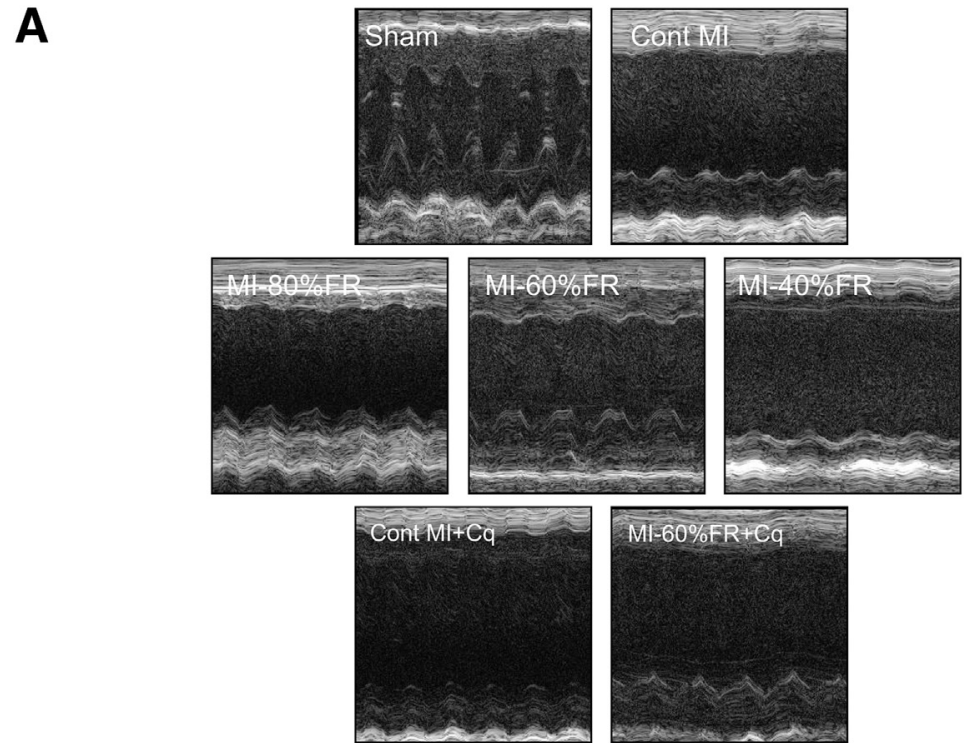

B
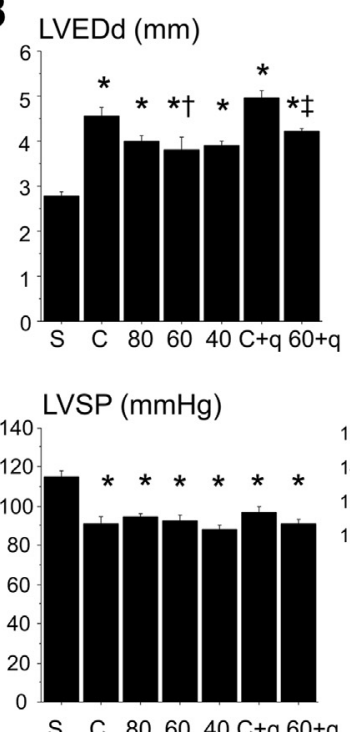
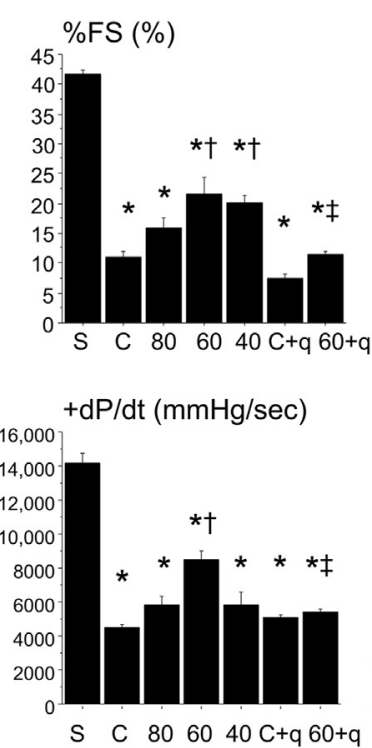
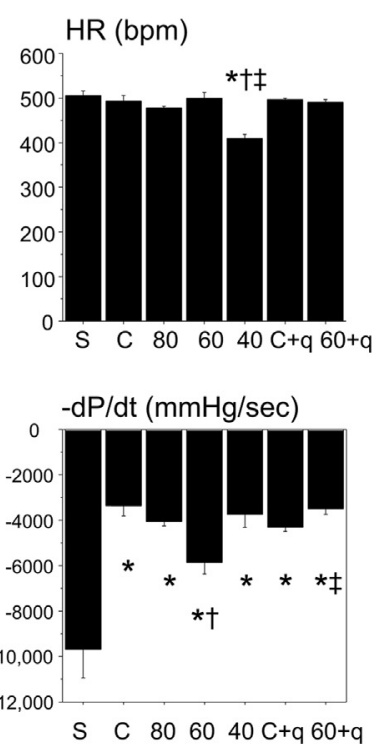

Figure 1 Effects of FR on cardiac remodeling and function evaluated using echocardiography and cardiac catheterization at 3 weeks after infarction. A: Representative M-mode echocardiograms at the level of the ventricles in each group. B: Hemodynamic parameters. Data are expressed as means \pm SEM. $n=6$ mice per group. ${ }^{*} P<0.05$ versus sham surgery. ${ }^{\dagger} P<0.05$ versus control. ${ }^{\ddagger} P<0.05$ versus $60 \%$ FR. bpm, beats per minute; $C$ or Cont, control; $\mathrm{Cq}$ or $\mathrm{q}$, chloroquine; $\mathrm{dP} / \mathrm{dt}$, change in pressure over time in the left ventricle; $\% F S$, percent fractional shortening; HR, heart rate; LVEDd, left ventricular end-diastolic dimension; LVSP, left ventricular systolic pressure; MI, myocardial infarction; $S$, sham surgery; 40 or 60 or 80 , FR percentage. 

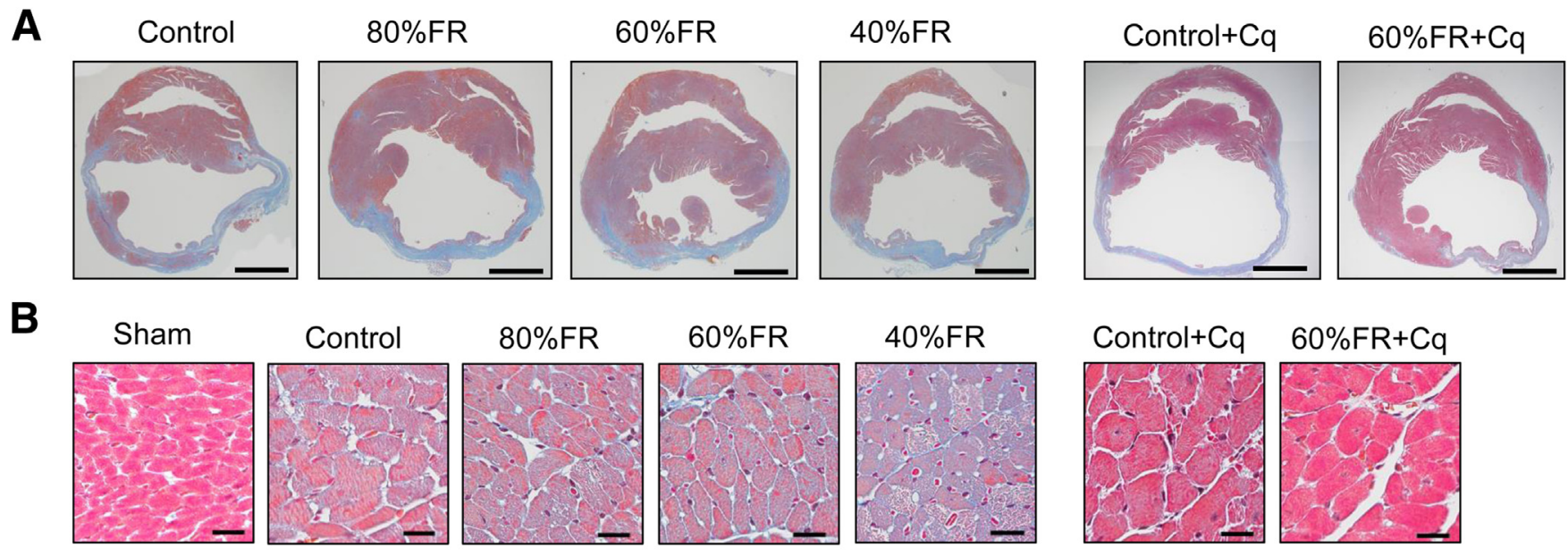

C
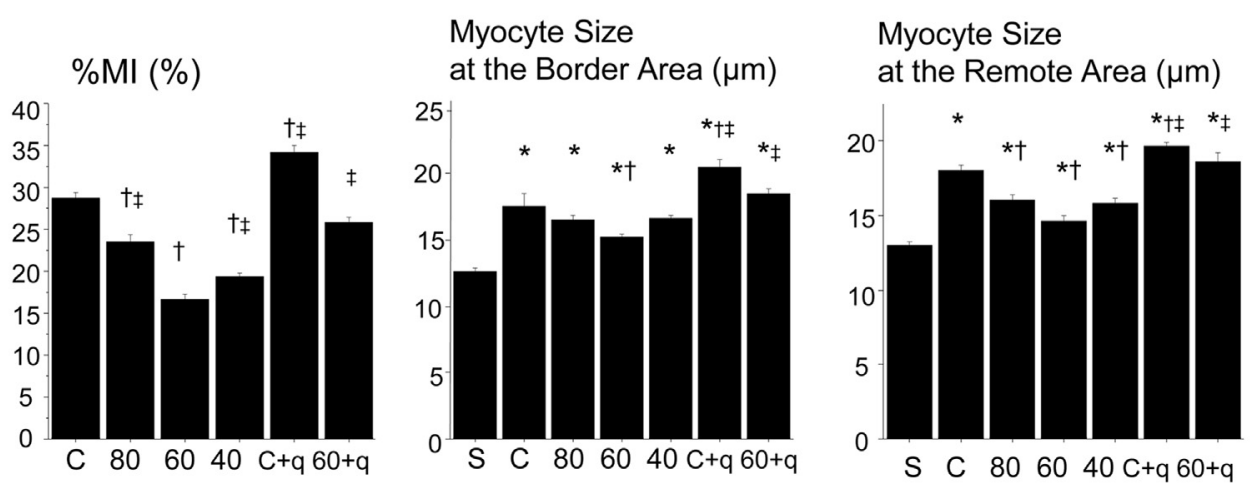

Figure 2 Histological evaluation of effects of FR on the postinfarction heart (Masson's trichrome stain). A: Ventricular transverse slices. B: Representative micrographs show size of cardiomyocytes in the remote area. C: Percent infarct size and cardiomyocyte size in the remote and border areas. Data are expressed as means \pm SEM. $n=6$ mice per group. ${ }^{*} P<0.05$ versus sham surgery. ${ }^{\dagger} P<0.05$ versus control. ${ }^{\ddagger} P<0.05$ versus $60 \%$ FR. Scale bars: 1 $\mathrm{mm}(\mathbf{A}) ; 10 \mu \mathrm{m}(\mathbf{B})$.

\section{Effect of FR on Autophagy in Postinfarction Hearts}

Immunohistochemistry revealed that the numbers of LC3positive autophagic vacuoles were significantly increased within surviving cardiomyocytes after infarction (Figure 3A). Immunopositive dots were distributed in cardiomyocytes situated both in areas bordering the infarction and in more remote areas. Within the infarct area, however, the dot number was extremely low. In hearts from mice undergoing FR, immunopositive dot numbers were significantly higher than control in both border and remote areas, and the magnitude of the increase was greater in the $40 \%$ and $60 \%$ FR groups than in the $80 \%$ FR group (Figure 3A).

Western blot analysis showed that expression of LC3-II was up-regulated in the postinfarction heart (Figure 3B). The LC3-II/LC3-I ratio was significantly increased, and was increased still further by FR, which is indicative of greater autophagic turnover. ${ }^{29}$ Cathepsin D, a lysosomal enzyme, was also up-regulated in postinfarction hearts, and this upregulation was also augmented by FR (Figure 3B). The LC3-binding protein 62 regulates the formation of protein aggregates and is removed in the final digestion step during autophagy. ${ }^{30}$ Consequently, an increase in p62 levels indicates an increase in protein aggregation or impairment of their digestion. Levels of p62 were significantly increased in the postinfarction heart, but were reduced by FR (Figure 3B).

Electron microscopic examination revealed that both autophagic vacuoles and lysosomes were abundant within cardiomyocytes in the postinfarction heart, and their numbers appeared to be further increased by FR (Figure 4). In addition, direct measurements revealed significantly increased myocardial ATP content in all FR groups, with the highest levels in the 60\% FR group (Figure 5). These results suggest that, through energy recycling, autophagy gives full play to cardiomyocyte function in mice undergoing FR.

\section{Effect of Autophagy Inhibition on the Benefits of FR in} Postinfarction Hearts

We next investigated effects of inhibiting autophagy using chloroquine on the beneficial effects of FR on postinfarction hearts. We also investigated several autophagy-related signals in this set of experiments. Treatment with chloroquine was started at 1 week after infarction and continued for 2 weeks in the control and the 60\% FR groups. 

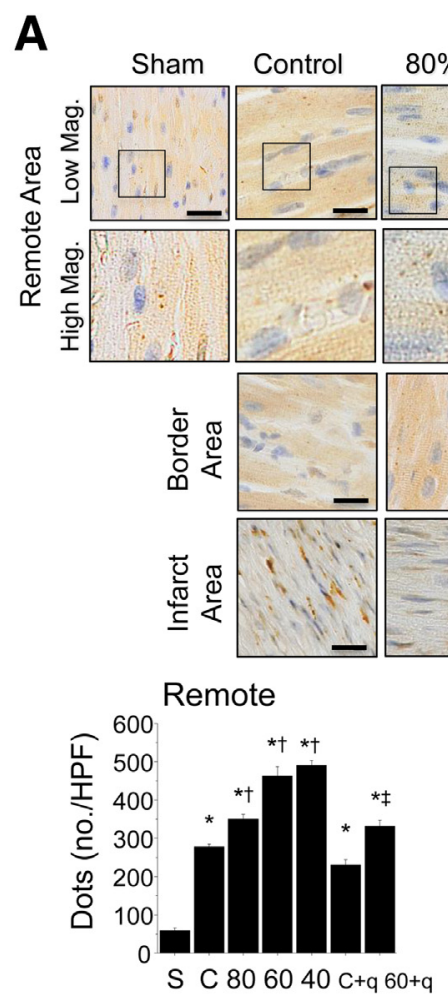
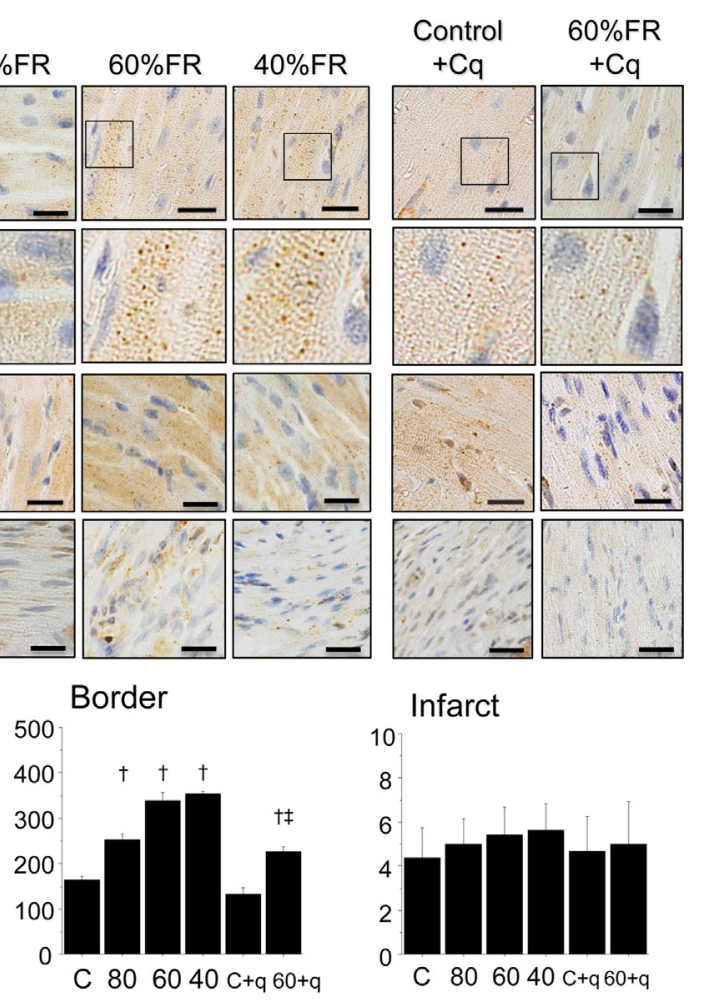

B

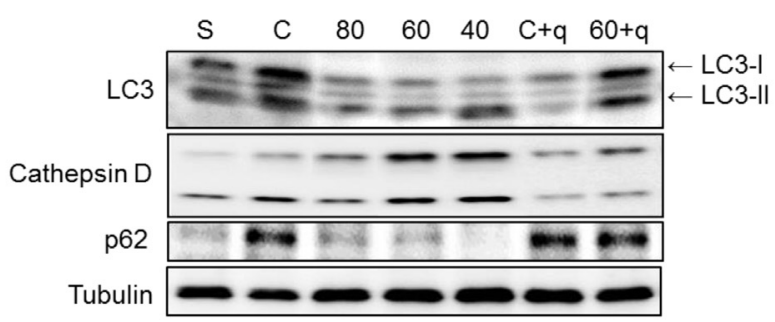

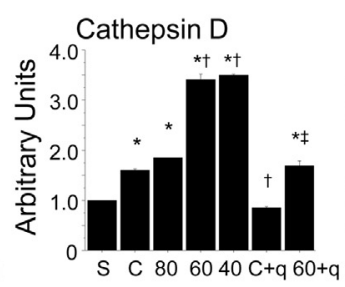

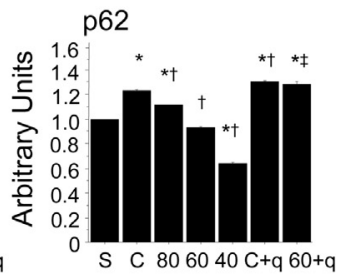

Figure 3 Postinfarction accumulation of autophagic vacuoles. A: Immunohistochemical labeling of LC3 (brown) in ventricular tissues in an area remote from the infarct, in tissue bordering the infarct, and within the infarct area. For the remote area, the LC3 immunopositive dots can be seen clearly in the high-magnification images, which correspond to the boxed regions in the adjacent low-magnification image. LC3 immunopositive dots per HPF $(\times 600)$ were quantified in the remote, border, and infarct areas. B: Western blots of the autophagy-related proteins LC3, cathepsin D, and p62 in heart. The LC3-II/LC3-I ratios were calculated and the intensity of the cathepsin D and p62 bands were quantified. Data are expressed as means \pm SEM. $n=6$ mice per group. ${ }^{*} P<0.05$ versus sham surgery. ${ }^{\dagger} P<0.05$ versus control. ${ }^{\ddagger} P<0.05$ versus $60 \%$ FR. Scale bar $=20 \mu \mathrm{m}$.

\section{Cardiac Function and Pathology}

Chloroquine treatment exacerbated postinfarction cardiac dysfunction and remodeling in both the control and the $60 \%$ FR groups; in the latter, chloroquine appeared to cancel the benefits of a restricted diet (Figures 1 and 2).

\section{Cardiac Autophagy}

The increase in LC3 immunopositive dots normally seen in cardiomyocytes from mice in the $60 \%$ FR group was diminished by treatment with chloroquine (Figure $3 \mathrm{~A}$ ). Expression of both LC3-II and LC3-I was down-regulated in chloroquine-treated hearts, and thus the LC3-II/LC3-I ratio was significantly reduced (Figure 3B). The level of cathepsin $\mathrm{D}$ was also markedly reduced by chloroquine
(Figure 3B), and the postinfarction reduction in p62 induced by FR was inhibited by chloroquine (Figure 3B). Ultrastructurally, the surviving cardiomyocytes in the chloroquine-treated hearts lacked indicators of autophagic activation, such as abundant lysosomes and autophagosomes (Figure 4). Chloroquine treatment also reduced postinfarction myocardial ATP in control hearts (Figure 5) and significantly attenuated the increase in myocardial ATP normally induced by $60 \%$ FR.

\section{Autophagy-Related Signals}

We next examined autophagy-related signal transduction in postinfarction hearts with or without $60 \%$ FR and/or chloroquine interventions. AMPK belongs to a conserved 


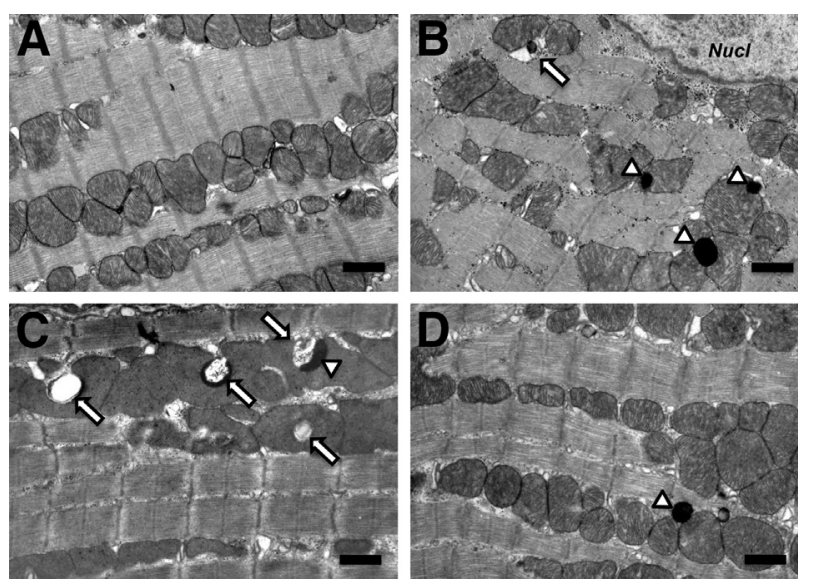

Figure 4 Electron micrographs show autophagic vacuoles (arrows) and lysosomes (arrowheads) in cardiomyocytes of sham-operated heart (A), postinfarction control heart (B), postinfarction heart treated with $60 \% \mathrm{FR}$ (C), and postinfarction heart simultaneously treated with $60 \%$ FR and chloroquine (D). Nucl, nucleus. Scale bar $=1 \mu \mathrm{m}$.

family of protein kinases that are activated by ATP depletion and the resultant accumulation of AMP, and it is an important regulator of autophagy stimulated by cellular starvation. ${ }^{31}$ A significant increase in the level of activated (phosphorylated) AMPK was observed in the postinfarction heart, and the level was increased still further by $60 \%$ FR or chloroquine (Figure 6A). A recent study found that direct activation (phosphorylation) of the mammalian autophagy-initiating kinase ULK1, a homolog of yeast Atg1, by AMPK is necessary for autophagy induction. ${ }^{32}$ As expected, ULK1 activation (based on the myocardial levels of p-ULK1) was significantly increased by FR (Figure 6A).

We also assessed the postinfarction activity of Akt, a key prosurvival molecule that negatively regulates myocardial AMPK activity. ${ }^{33}$ Western blot analysis showed that levels of p-Akt were significantly higher in postinfarction hearts than in sham-operated hearts, but they were unaffected by any of the treatments in this study (Figure 6B). This suggests that, in the present model, FR acts directly or indirectly on AMPK without affecting the Akt pathway.

Sirt1 (a NAD ${ }^{+}$-dependent protein deacetylase and a mammalian ortholog of yeast Sir2) deacetylates histone polypeptides, with a preference for histone $\mathrm{H} 3$ lysine $9 .{ }^{34}$ Sirt1 levels were similar in all of the treatment groups (Figure 6C). On the other hand, the level of acetylhistone, which is inversely related to Sirt1 activity, was significantly increased in the infarcted heart. The increase in acetyl-histone (reflecting inactivity of Sirt1) was reversed by FR but was unaffected by chloroquine (Figure 6C). Myocardial Sirt1 thus appears to be activated by FR in the infarcted heart, but the lack of effect of chloroquine suggests that Sirt1 is not involved in the autophagic response.

\section{Autophagy-Related Signal Transduction in Cultured Cardiomyocytes}

Last, we used cultured cardiomyocytes to investigate the roles played by AMPK and ULK1 in the mechanism underlying FR-induced autophagy. Isolated neonatal mouse cardiomyocytes were treated with glucose-free medium or chloroquine with or without simultaneous treatment with the AMPK inhibitor compound C for 4 hours. Glucose depletion brought about an increase in p-AMPK and p-ULK1 levels and an increase in the numbers of LC3 immunopositive dots (indicating autophagosomes and autolysosomes) observed within cardiomyocytes, but treatment with compound C completely canceled those effects (Figure 7, A and B). The ATP content in cardiomyocytes was increased by glucose depletion, and this increase was inhibited by compound C (Figure 7C). Treatment with chloroquine also led to an increase in p-AMPK and p-ULK1 levels, and an increase in LC3 immunopositive dots, but a marked reduction in the ATP content of cardiomyocytes, none of which were affected by simultaneous treatment with compound $\mathrm{C}$ (Figure 7, A-C). Taken together, these results indicate that glucose depletion enhances autophagy via an AMPK-ULK1 pathway, and that glucose depletion and chloroquine regulate AMPK activity and downstream events in distinctly different ways, although both activate AMPK. We suggest that glucose depletion directly activates AMPK to promote autophagy, because autophagy evoked by glucose depletion is inhibited by the AMPK inhibitor. Chloroquine likely inhibits autophagy by interfering with fusion between autophagosomes and lysosomes, which results in a reduction in cellular ATP content and in turn stimulates AMPK activity (Figure 7D).

\section{Discussion}

\section{Mechanisms for the Benefits of FR on the} Postinfarction Heart

The main finding of the present study is that restriction of food intake after a large myocardial infarction, starting during the subacute stage, significantly mitigates the adverse

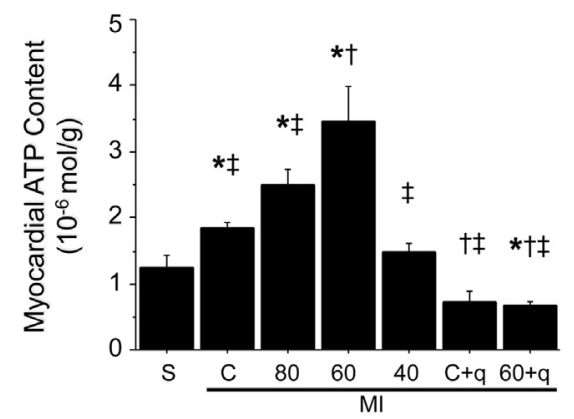

Figure 5 Myocardial ATP content. Data are expressed as means \pm SEM. $n=6$ mice per group. ${ }^{\star} P<0.05$ versus sham surgery. ${ }^{\dagger} P<0.05$ versus control. ${ }^{\ddagger} P<0.05$ versus $60 \%$ FR. 
A

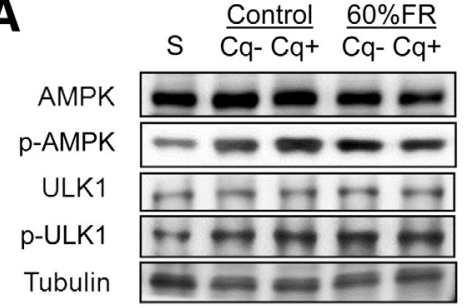

B

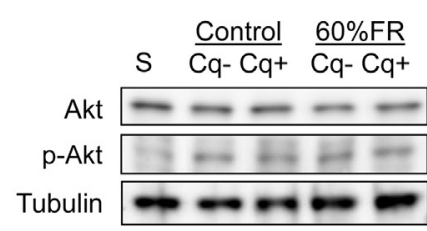

C

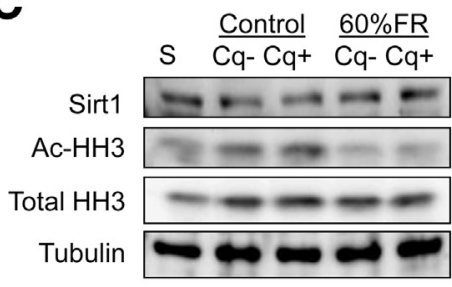

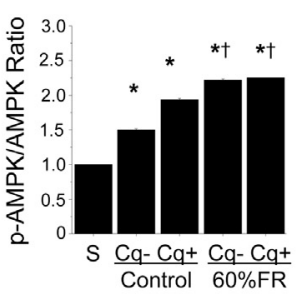
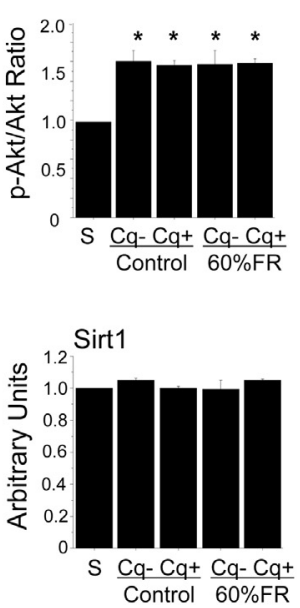

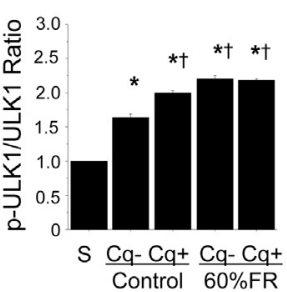

Figure 6 Western blots and densitometric analysis of the autophagy-related proteins AMPK, ULK1, Akt, and Sirt1. A: AMPK, p-AMPK, ULK1, and p-ULK1. B: Akt and p-Akt. C: Sirt1, acetylated histone $\mathrm{H} 3$, and total histone H3. Data are expressed as means \pm SEM. $n=6$ mice per group. ${ }^{*} P<0.05$ versus sham surgery. ${ }^{\dagger} P<0.05$ versus control. ${ }^{\ddagger} P<0.05$ versus $60 \%$ FR.

left ventricular remodeling and subsequent heart failure seen at the chronic stage. The therapeutic efficiency was strongest in mice receiving $60 \%$ of their normal food intake. We suggest that these beneficial effects of FR mainly reflect the ability of the heart to increase autophagic activity, because the autophagy inhibitor chloroquine produced opposite outcomes when administered alone and offset the effects of FR when administered under those conditions. In addition, autophagic activity, myocardial ATP content, and the degree of improvement in left ventricular remodeling and function all varied in parallel with the extent of FR up to $60 \%$, supporting the notion that increases in autophagy induced by FR accelerate energy recycling to the benefit of postinfarction processes in the heart. At $40 \%$, however, FR failed to improve postinfarction left ventricular function and remodeling, despite marked up-regulation of autophagy. In this extreme case, myocardial ATP content was paradoxically reduced. Apparently, even marked up-regulation of autophagy could not compensate for the lack of ATP if FR was too severe to supply sufficient energy.

The accumulation of autophagic vacuoles can represent either increased formation or impaired digestion. In the present study, we confirmed an increase in the LC3-II/LC3-I ratio, which is an established indicator of autophagic turnover. ${ }^{29}$ Moreover, to examine autophagic flux in vivo, we used chloroquine alone and in combination with FR. Chloroquine inhibits autophagosome-lysosome fusion, thereby preventing the final digestion step in autophagy, and it is frequently used as an autophagy inhibitor. ${ }^{25}$ In postinfarction hearts, chloroquine suppressed autophagic activity and

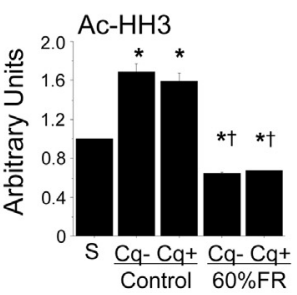

reduced numbers of autophagic vacuoles and LC3-II/LC3-I ratios, both of which were augmented by FR. These findings indicate that FR increases autophagic turnover or flux in failing hearts with old myocardial infarctions.

We recently studied the time course of autophagy in surviving cardiomyocytes during the 3 weeks after myocardial infarction and found that autophagy was very actively induced in remote areas and in border areas in the myocardium, and that activity in the remote areas was stronger during more chronic stages ( 2 and 3 weeks after infarction). ${ }^{15}$ The present study confirmed strong autophagic activity in remote areas at 3 weeks after infarction. With progression of cardiac remodeling, wall stress increases in parallel with ventricular dilatation, in accordance with Laplace's law. This causes tissue hypoxia, even in the remote myocardium, which may contribute to the induction of autophagy in failing hearts with large old infarctions.

\section{Autophagy-Related Molecular Signals Evoked by FR}

Energy recycling is one of the most important functions of autophagy, and we previously reported that autophagy increases myocardial ATP levels, leading to improved cardiac performance and cardiomyocyte survival. ${ }^{19}$ AMPK belongs to a conserved family of protein kinases and serves as a general integrator of the metabolic response to changes in energy availability; it is activated by ATP depletion and concomitant AMP accumulation (increases in the AMP/ ATP ratio). ${ }^{31,35}$ Here, we observed that more strict FR induced higher levels of AMPK activity. We also observed 
A

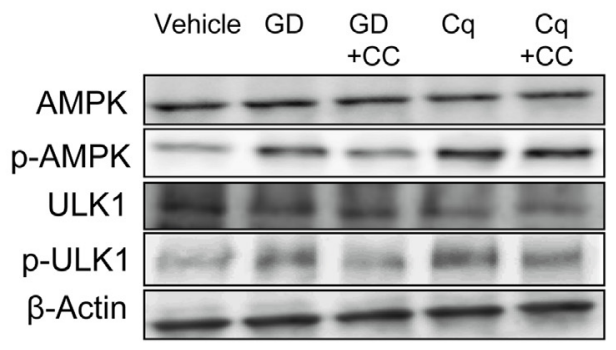



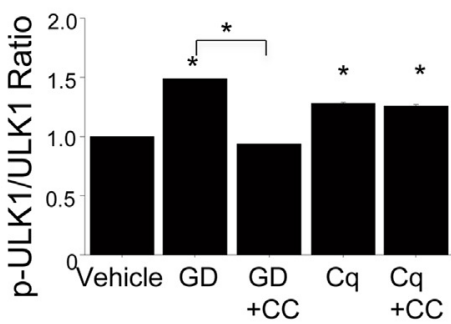

B
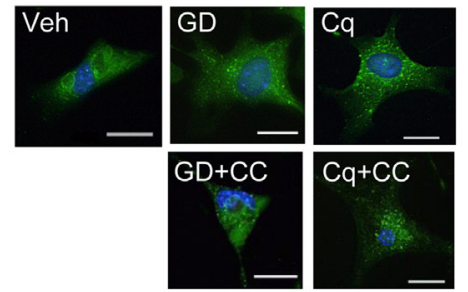

D

C

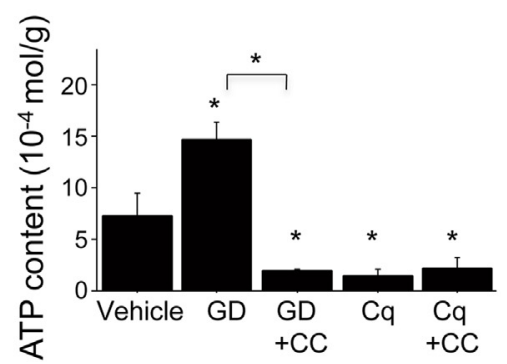

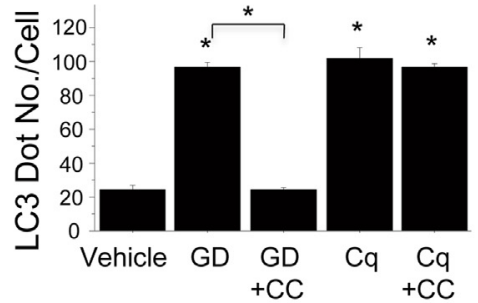

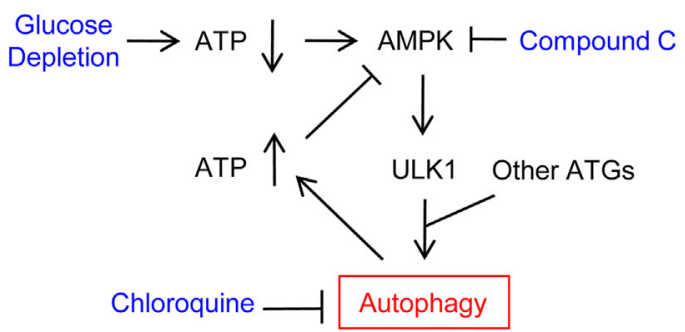

Figure 7 In vitro experiments using cultured mouse neonatal cardiomyocytes. A: Western blots with densitometric analysis of AMPK, p-AMPK, ULK1, and p-ULK1. B: Immunofluorescent labeling of LC3 appears as green dots in cardiomyocytes. C: ATP content of cardiomyocytes. D: Hypothesized signal transduction of autophagy affected by glucose depletion and chloroquine. Data are expressed as means \pm SEM. $n=3$ mice per group. ${ }^{*} P<0.05$ versus vehicle-treated group or between bracketed groups. Scale bar $=20$ $\mu \mathrm{m}$. ATGs, autophagy-related gene products; CC, compound C; GD, glucose depletion; Veh, vehicle. up-regulation of p-AMPK in chloroquine-treated hearts, which is consistent with our earlier finding that inhibition of autophagy by bafilomycin A1 interferes with the supply of ATP to the ischemic myocardium, leading to AMPK activation. ${ }^{15}$ The present in vitro experiments suggest that both glucose depletion and chloroquine stimulate AMPK activation. Glucose depletion directly activates AMPK to promote autophagy, whereas chloroquine acts indirectly. By inhibiting autophagy (most likely by interfering with the fusion between autophagosomes and lysosomes ${ }^{23-25}$ ), chloroquine reduces ATP levels, which in turn stimulates AMPK activity (Figure 7D). This is reminiscent of previously reported mechanisms regarding the beneficial effect of resveratrol on postinfarction cardiac remodeling. ${ }^{36}$

It is thought that AMPK can trigger autophagy through an indirect mechanism whereby it inhibits the activity of mTOR complex 1 by phosphorylating tuberous sclerosis type 2 (TSC2) and regulatory-associated protein of mTOR (Raptor). ${ }^{31,37}$ However, recent findings suggest a more direct mechanism whereby AMPK regulates autophagy through phosphorylation of ULK1 (mammalian homolog of yeast Atg1). ${ }^{38,39}$ The ULK1 kinase complex plays a central role in the induction of autophagy by regulating the earliest step in autophagosome formation. In the present study, we observed increases in the levels of both p-AMPK and
p-ULK1 in the postinfarction heart, which were further increased by FR. This suggests that AMPK activation and subsequent ULK1 activation represent one possible pathway to improved autophagic flux through FR (Figure 7D).

Although we confirmed that FR activates Sirt1, it remains unclear whether Sirt1 has a direct effect on autophagy. Sirt1 activity was maintained in the postinfarction heart despite treatment with chloroquine (Figure 6C). That chloroquine exacerbated left ventricular remodeling and dysfunction without affecting Sirt1 activity implies a dissociation between Sirt1 activity and cardioprotection. Nadtochiy et al ${ }^{40}$ reported that overexpression of Sirt1 protected against ischemia-reperfusion injury through stimulation of autophagy, but Kawashima et $\mathrm{al}^{41}$ reported that constitutive Sirt1 overexpression impaired mitochondrial function and reduced autophagy, resulting in cardiac dysfunction. Further study would be required to define the role of Sirt1 in postinfarction cardiac remodeling.

\section{Conclusions and Clinical Implications}

Food restriction enhances cardiomyocyte autophagy and appears to mitigate postinfarction cardiac remodeling and dysfunction. In mice, the optimal caloric restriction is to 
$60 \%$ of control. The clinical implication is that a dietary protocol started during the subacute stage of myocardial infarction could be a preventive strategy against progression of postinfarction left ventricular remodeling and heart failure.

\section{Acknowledgments}

We thank Kanami Aoki, Hiroe Inoue, Nao Kawauchi, Rei Banba, and Yui Fujii (Kyoto Women's University) for their technical assistance.

\section{References}

1. Pfeffer MA: Left ventricular remodeling after acute myocardial infarction. Annu Rev Med 1995, 46:455-466

2. Reimer KA, Vander Heide RS, Richard VJ: Reperfusion in acute myocardial infarction: effect of timing and modulating factors in experimental models. Am J Cardiol 1993, 72:13G-21G

3. Shan K, Kurrelmeyer K, Seta Y, Wang F, Dibbs Z, Deswal A, LeeJackson D, Mann DL: The role of cytokines in disease progression in heart failure. Curr Opin Cardiol 1997, 12:218-223

4. Weisman HF, Bush DE, Mannisi JA, Weisfeldt ML, Healy B: Cellular mechanisms of myocardial infarct expansion. Circulation 1988, 78: 186-201

5. Elsässer A, Schlepper M, Klövekorn WP, Cai WJ, Zimmermann R, Müller KD, Strasser R, Kostin S, Gagel C, Münkel B, Schaper W, Schaper J: Hibernating myocardium: an incomplete adaptation to ischemia. Circulation 1997, 96:2920-2931

6. Elsässer A, Vogt AM, Nef H, Kostin S, Möllmann H, Skwara W, Bode C, Hamm C, Schaper J: Human hibernating myocardium is jeopardized by apoptotic and autophagic cell death. J Am Coll Cardiol 2004, 43:2191-2199

7. Yan L, Vatner DE, Kim SJ, Ge H, Masurekar M, Massover WH, Yang G, Matsui Y, Sadoshima J, Vatner SF: Autophagy in chronically ischemic myocardium. Proc Natl Acad Sci USA 2005, 102: 13807-13812

8. Shintani T, Klionsky DJ: Autophagy in health and disease: a doubleedged sword. Science 2004, 306:990-995

9. Clarke PG: Developmental cell death: morphological diversity and multiple mechanisms. Anat Embryol 1990, 181:195-213

10. Nakai A, Yamaguchi O, Takeda T, Higuchi Y, Hikoso S, Taniike M, Omiya S, Mizote I, Matsumura Y, Asahi M, Nishida K, Hori M, Mizushima N, Otsu K: The role of autophagy in cardiomyocytes in the basal state and in response to hemodynamic stress. Nat Med 2007, 13: 619-624

11. Matsui Y, Takagi H, Qu X, Abdellatif M, Sakoda H, Asano T, Levine B, Sadoshima J: Distinct roles of autophagy in the heart during ischemia and reperfusion: roles of AMP-activated protein kinase and Beclin 1 in mediating autophagy. Circ Res 2007, 100:914-922

12. Takemura G, Miyata S, Kawase Y, Okada H, Maruyama R, Fujiwara H: Autophagic degeneration and death of cardiomyocytes in heart failure. Autophagy 2006, 2:212-214

13. Hein S, Arnon E, Kostin S, Schönburg M, Elsässer A, Polyakova V, Bauer EP, Klövekorn WP, Schaper J: Progression from compensated hypertrophy to failure in the pressure-overloaded human heart: structural deterioration and compensatory mechanisms. Circulation 2003, 107:984-991

14. Gustafsson AB, Gottlieb RA: Autophagy in ischemic heart disease. Circ Res 2009, 104:150-158

15. Kanamori H, Takemura G, Goto K, Maruyama R, Tsujimoto A, Ogino A, Takeyama T, Kawaguchi T, Watanabe T, Fujiwara T, Fujiwara H,
Seishima M, Minatoguchi S: The role of autophagy emerging in postinfarction cardiac remodelling. Cardiovasc Res 2011, 91:330-339

16. Blommaart EF, Luiken JJ, Blommaart PJ, van Woerkom GM, Meijer AJ: Phosphorylation of ribosomal protein S6 is inhibitory for autophagy in isolated rat hepatocytes. J Biol Chem 1995, 270: $2320-2326$

17. Schmelzle T, Hall MN: TOR, a central controller of cell growth. Cell 2000, 103:253-262

18. Kuma A, Hatano M, Matsui M, Yamamoto A, Nakaya H, Yoshimori T, Ohsumi Y, Tokuhisa T, Mizushima N: The role of autophagy during the early neonatal starvation period. Nature 2004, 432:1032-1036

19. Kanamori H, Takemura G, Maruyama R, Goto K, Tsujimoto A, Ogino A, Li L, Kawamura I, Takeyama T, Kawaguchi T, Nagashima K, Fujiwara T, Fujiwara H, Seishima M, Minatoguchi S: Functional significance and morphological characterization of starvation-induced autophagy in the adult heart. Am J Pathol 2009, 174:1705-1714

20. Wohlgemuth SE, Julian D, Akin DE, Fried J, Toscano K, Leeuwenburgh C, Dunn WA Jr: Autophagy in the heart and liver during normal aging and calorie restriction. Rejuvenation Res 2007, 10:281-292

21. Kanamori H, Takemura G, Li Y, Okada H, Maruyama R, Aoyama T, Miyata S, Esaki M, Ogino A, Nakagawa M, Ushikoshi $H$, Kawasaki M, Minatoguchi S, Fujiwara H: Inhibition of Fas-associated apoptosis in granulation tissue cells accompanies attenuation of postinfarction left ventricular remodeling by olmesartan. Am J Physiol Heart Circ Physiol 2007, 292:H2184-H2194

22. Li Y, Takemura G, Kosai K, Takahashi T, Okada H, Miyata S, Yuge K, Nagano S, Esaki M, Khai NC, Goto K, Mikami A, Maruyama R, Minatoguchi S, Fujiwara T, Fujiwara H: Critical roles for the Fas/Fas ligand system in postinfarction ventricular remodeling and heart failure. Circ Res 2004, 95:627-636

23. Ohkuma S, Poole B: Fluorescence probe measurement of the intralysosomal $\mathrm{pH}$ in living cells and the perturbation of $\mathrm{pH}$ by various agents. Proc Natl Acad Sci USA 1978, 75:3327-3331

24. Sewell RB, Barham SS, LaRusso NF: Effect of chloroquine on the form and function of hepatocyte lysosomes. Morphologic modifications and physiologic alternations related to the biliary excretion of lipids and proteins. Gastroenterology 1983, 85:1146-1153

25. Iwai-Kanai E, Yuan H, Huang C, Sayen MR, Perry-Garza CN, Kim L, Gottlieb RA: A method to measure cardiac autophagic flux in vivo. Autophagy 2008, 4:322-329

26. Wang L, Feng ZP, Kondo CS, Sheldon RS, Duff HJ: Developmental changes in the delayed rectifier $\mathrm{K}+$ channels in mouse heart. Circ Res 1996, 79:79-85

27. Chiamvimonvat N, Kargacin ME, Clark RB, Duff HJ: Effects of intracellular calcium on sodium current density in cultured neonatal rat cardiac myocytes. J Physiol 1995, 483:307-318

28. Lee M, Hwang JT, Lee HJ, Jung SN, Kang I, Chi SG, Kim SS, Ha J: AMPactivated protein kinase activity is critical for hypoxia-inducible factor-1 transcriptional activity and its target gene expression under hypoxic conditions in DU145 cells. J Biol Chem 2003, 278:39653-39661

29. Kabeya Y, Mizushima N, Ueno T, Yamamoto A, Kirisako T, Noda T, Kominami E, Ohsumi Y, Yoshimori T: LC3, a mammalian homologue of yeast Apg8p, is localized in autophagosome membranes after processing [Erratum appeared in EMBO J 2003, 22:4577]. EMBO J 2000, 19:5720-5728

30. Komatsu M, Waguri S, Koike M, Sou YS, Ueno T, Hara T, Mizushima N, Iwata J, Ezaki J, Murata S, Hamazaki J, Nishito Y, Iemura S, Natume T, Yanagawa T, Uwayama J, Warabi E, Yoshida H, Ishii T, Kobayashi A, Yamamoto M, Yue Z, Uchiyama Y, Kominami E, Tanaka K: Homeostatic levels of p62 control cytoplasmic inclusion body formation in autophagy-deficient mice. Cell 2007, 131:1149-1163

31. Inoki K, Zhu T, Guan KL: TSC2 mediates cellular energy response to control cell growth and survival. Cell 2003, 115:577-590 
32. Lee JW, Park S, Takahashi Y, Wang HG: The association of AMPK with ULK1 regulates autophagy. PLoS One 2010, 5:e15394

33. Kovacic S, Soltys CL, Barr AJ, Shiojima I, Walsh K, Dyck JR: Akt activity negatively regulates phosphorylation of AMP-activated protein kinase in the heart [Erratum appeared in J Biol Chem 2004, 279:2332]. J Biol Chem 2003, 278:39422-39427

34. Vaquero A, Scher M, Lee D, Erdjument-Bromage H, Tempst P, Reinberg D: Human SirT1 interacts with histone H1 and promotes formation of facultative heterochromatin. Mol Cell 2004, 16:93-105

35. Samari HR, Seglen PO: Inhibition of hepatocytic autophagy by adenosine, aminoimidazole-4-carboxamide riboside, and N6mercaptopurine riboside. Evidence for involvement of AMPactivated protein kinase. J Biol Chem 1998, 273:23758-23763

36. Kanamori H, Takemura G, Goto K, Tsujimoto A, Ogino A, Takeyama T, Kawaguchi T, Watanabe T, Morishita K, Kawasaki M, Mikami A, Fujiwara T, Fujiwara H, Seishima M, Minatoguchi S: Resveratrol reverses remodeling in hearts with large, old myocardial infarctions through enhanced autophagy-activating AMP kinase pathway. Am J Pathol 2013, 182:701-713
37. Gwinn DM, Shackelford DB, Egan DF, Mihaylova MM, Mery A, Vasquez DS, Turk BE, Shaw RJ: AMPK phosphorylation of raptor mediates a metabolic checkpoint. Mol Cell 2008, 30:214-226

38. Egan DF, Shackelford DB, Mihaylova MM, Gelino S, Kohnz RA, Mair W, Vasquez DS, Joshi A, Gwinn DM, Taylor R, Asara JM, Fitzpatrick J, Dillin A, Viollet B, Kundu M, Hansen M, Shaw RJ: Phosphorylation of ULK1 (hATG1) by AMP-activated protein kinase connects energy sensing to mitophagy. Science 2011, 331:456-461

39. Kim J, Kundu M, Viollet B, Guan KL: AMPK and mTOR regulate autophagy through direct phosphorylation of Ulk1. Nat Cell Biol 2011, 13:132-141

40. Nadtochiy SM, Yao H, McBurney MW, Gu W, Guarente L, Raham I, Brookes PS: SIRT1-mediated acute cardioprotection. Am J Physiol Heart Circ Physiol 2011, 301:H1506-H1512

41. Kawashima T, Inuzuka Y, Okuda J, Kato T, Niizuma S, Tamaki Y, Iwanaga Y, Kawamoto A, Narazaki M, Matsuda T, Adachi S, Takemura G, Kita T, Kimura T, Shioi T: Constitutive SIRT1 overexpression impairs mitochondria and reduces cardiac function in mice. J Mol Cell Cardiol 2011, 51:1026-1036 